\title{
Oltre il pregiudizio: la specificità dei processi di sviluppo delle famiglie contemporanee
}

\author{
Laura Fruggeri \\ Già professore ordinario di Psicologia delle relazioni familiari presso I'Università di Parma, è direttrice della Scuola \\ di psicoterapia sistemico-costruzionista-dialogica del Centro bolognese di terapia della famiglia, Centro bolognese \\ di terapia della famiglia
}

\begin{abstract}
Le famiglie tra diversità e discontinuità I mutamenti demografici, legislativi, socio-culturali e tecnologici che negli ultimi anni hanno caratterizzato anche la società italiana si sono riverberati sulle famiglie, con sostanziali e irreversibili ripercussioni sui modi di vivere i rapporti affettivi e i legami di cura nella quotidianità.
\end{abstract}

La legge sul divorzio ha cambiato la struttura delle famiglie. Infatti, le famiglie nucleari tradizionali hanno gemmato le famiglie post separazione, alcune delle quali, a loro volta, si sono trasformate in famiglie ricomposte in cui la coppia coniugale si forma avendo già la responsabilità genitoriale di figli nati da precedenti unioni. Così, a fianco delle tradizionali famiglie nucleari (nelle quali: 1 . le stesse persone negoziano tra loro sia il modo di essere genitori sia quello di essere partner; 2 . i confini simbolici della rete dei rapporti affettivi coincidono con i confini fisico-spaziali del gruppo coabitante; 3 . i procreatori sono le stesse persone che provvedono alla crescita e all'allevamento della prole), emergono nuove forme familiari caratterizzate invece da discontinuità strutturali e da plurinuclearità. Nelle famiglie post separazione e in quelle ricomposte, infatti, la coppia genitoriale biologica non è coppia coniugale o è diversa da quella coniugale; inoltre a fianco e a sostegno di una genitorialità biologica può costituirsi una genitorialità socioaffettiva, quella cioè degli eventuali nuovi partner dei genitori separati; i confini affettivi e quelli spaziali non coincidono poiché la famiglia, come rete di legami affettivi e di cura, si articola attraverso diversi nuclei abitativi (ogni genitore separato con i figli e con eventuale nuovo partner) (Fruggeri, 2005).

Le trasformazioni strutturali delle famiglie hanno un'ormai stabile presenza e una chiara visibilità sia nella società, sia nella ricerca scientifica. Gli studi sul funzionamento delle famiglie con genitori separati e di quelle ricomposte, e quelli che ne hanno valutato gli effetti sul benessere socioemotivo dei figli risalgono agli anni Ot- tanta e Novanta. Tutte queste ricerche hanno dimostrato che crescere in queste famiglie non comporta più rischi di quanto non ne comporti crescere in famiglie con genitori uniti. Le indagini, infatti, hanno dimostrato in modo consistente e metodologicamente fondato che "non è la forma (o struttura) delle famiglie che incide sul benessere dei suoi membri, bensì la qualità delle relazioni e dei processi che prendono corpo nel gruppo" (Golombok, 2000; Parke, 2013).

Tuttavia, occorre riconoscere che le caratteristiche strutturali di queste famiglie comportano dei compiti specifici:

1 Le famiglie derivate dalla riorganizzazione di quelle nucleari tradizionali si formano in seguito a rotture, separazioni, abbandoni o ricomposizioni; per questo si trovano ad affrontare l'elaborazione di perdite, allontanamenti, fratture e a impegnarsi nella gestione collaborativa dei conflitti.

2 Essendo articolate per nuclei abitativi diversi, le famiglie post separazione e quelle ricomposte, vivono la quotidianità oltre lo spazio condiviso. Le strategie attraverso cui ogni membro mantiene e alimenta, giorno dopo giorno, il proprio rapporto con gli altri è frutto di una coordinazione e di una negoziazione di modi, tempi e spazi, che si fondano sull'intento da parte degli adulti di favorire la possibilità per i figli di mantenere e godere del rapporto con tutti, anche attraversando i confini spaziali che delimitano i nuclei abitativi. La non coabitazione deve essere compensata da una comunicazione fluida e costante tra tutti coloro che hanno la responsabilità genitoriale.

3 I nuclei biologici e abitativi che compongo le famiglie plurinucleari sono tra loro intrecciati, intersecati e interdipendenti. Per questo, ciò che avviene in un nucleo, la qualità delle relazioni tra i membri, i loro progetti futuri non prescindono mai da ciò che avviene in altri nuclei, dalla qualità delle relazioni che si sviluppano in essi e dalla progettualità che li orienta.

4 Nelle famiglie ricomposte, i partner degli ex coniugi sono figure genitoriali di complemento che devono trovare uno spazio relazionale-educativo. Non essendoci ancora modelli di riferimento sociali codificati, il ruolo del genitore acquisito è affidato alla negoziazione quotidiana dei membri della famiglia.

5 Le famiglie plurinucleari devono saper gestire con flessibilità i confini per favorire la pluriappartenenza che si accompagna alla loro struttura plurinucleare, evitando di mettere i membri in una dolorosa situazione di conflitto di lealtà.

Si tratta di compiti complessi, per assolvere i quali le famiglie devono poter contare sul sostegno del contesto sociale e in particolare dei professionisti dei servizi che accompagnano le famiglie nei loro processi, i quali, per questo, ne devono conoscere la specificità.

Oltre ai cambiamenti strutturali, altre trasformazioni hanno investito le famiglie negli ultimi anni.

La rivoluzione femminista, i movimenti libertari, lo sviluppo delle biotecnologie e la legislazione che riconosce filiazioni adottive hanno introdotto nuovi modi di fondazione delle famiglie che sono diverse dalle famiglie nucleari tradizionali non per la loro struttura, ma per come si originano. Sono le famiglie che si formano grazie alle tecniche di riproduzione assistita (con coppie etero e omosessuali), le famiglie con madre single per scelta, le famiglie adottive. Si tratta di famiglie che hanno una struttura nucleare e che, dunque, non devono affrontare i compiti attinenti alla plurinuclearità illustrati nel paragrafo precedente. Esse sono chiamate ad affrontare altre questioni: quelle concernenti la narrazione del come sono diventate "famiglia”. Infatti, in esse, la genitorialità è resa possibile grazie al contributo di un "terzo", che può assumere diverse fisionomie a seconda della procedura utilizzata per l'atti- 
vazione della genitorialità. Nell'inseminazione omologa, il bambino condivide con entrambi i genitori il patrimonio genetico, ma la sua nascita è stata resa possibile dal ricorso alla tecnologia; nelle altre procedure (donazione di seme o di ovociti e gestazione per altri), il bambino condivide con un solo genitore il patrimonio genetico e la sua nascita è stata resa possibile dal contributo di una terza persona; il figlio adottivo non condivide con nessuno dei genitori il patrimonio genetico e la sua filiazione è avvenuta grazie ad altri genitori biologici.

Anche l'indagine scientifica sui processi evolutivi e sugli esiti dello sviluppo dei membri delle famiglie a diversa fondazione, per quanto fenomeno più recente, può contare ormai su un robusto insieme di dati e di risultati che confermano come l'assenza di connessioni genetiche o gestionali tra genitori e figli non abbia alcun effetto negativo sulla qualità dei loro rapporti, né sul benessere dei figli (Golombok, 2015).

E tuttavia anche queste famiglie hanno compiti specifici da affrontare.

La condivisione con il/la bambino/a delle modalità del concepimento - e dunque del suo legame genetico con i genitori - e la collocazione nello scenario delle loro relazioni delle figure che hanno contribuito al concepimento (che però non condividono col bambino la vita quotidiana), diventano temi salienti in queste famiglie. La narrazione della nascita è centrale per chiunque; tuttavia, mentre la condizione della genitorialità fondata sul rapporto sessuale tra i genitori ha a disposizione un repertorio condiviso di storie, simboli, immagini, metafore; la genitorialità single, quella tecnicamente assistita e l'adozione, non possono contare su un così rassicurante ancoraggio culturale, e i genitori si trovano nella posizione di chi questi repertori narrativi li deve creare. Le indagini hanno dimostrato che questo rende il loro compito solo più difficile, non impossibile a priori. In generale, le ricerche mostrano che il buon adattamento dei figli che crescono in queste famiglie è anche legato al modo in cui gli adulti elaborano innanzitutto per se stessi i percorsi inconsueti attraverso cui sono diventati famiglia e sono così in grado di aiutare i propri figli a elaborarli a loro volta (Lingiardi, Carone, 2016). In questo senso,

- l'esplicitazione delle fantasie sul "terzo";

- la narrabilità delle origini inconsuete;

- la legittimazione a interrogarsi e a porre domande circa la non condivisione genetica tra genitori e figli;

- l'apertura alle informazioni;

diventano i puntelli di una comunicazione familiare che costruisce legami e consolida appartenenze. Questi percorsi di "tra- sparenza" nelle famiglie sono complessi e possono richiedere un sopporto professionale che tuttavia non può che partire dalla consapevolezza che si tratta di temi nuovi e dunque dalla acquisizione di conoscenze circa la specificità dei processi e delle dinamiche implicate.

Ma le famiglie d'oggi sono diverse per altre molteplici ragioni.

Gli epocali processi migratori, il diffondersi dei mezzi di comunicazione informatizzati, l'invecchiamento della popolazione hanno prodotto circostanze di vita, che pongono le famiglie davanti a sfide inedite: affrontare l'inserimento in un nuovo contesto culturale; gestire la presenza di malattie degenerative; vivere le relazioni a distanza; fare coppia e famiglia all'epoca degli smartphone e dei social network. Si tratta di circostanze di vita che mettono le famiglie nelle condizioni di gestire le "perdite ambigue", di vivere tra più culture, di "addomesticare" i prodotti del mondo digitale.

Pauline Boss (1999) ha elaborato il costrutto di perdita ambigua che bene descrive la condizione psicologica dei membri di certe famiglie sempre più diffuse anche nel contesto italiano. Lautrice identifica due tipi di perdita ambigua:

1 un membro della famiglia è fisicamente assente, ma psicologicamente presente perché vivo in un altrove da cui può ritornare o nel quale essere raggiunto (si pensi alle situazioni migratorie o all'incarcerazione);

2 un membro della famiglia è fisicamente presente, ma assente psicologicamente, emotivamente, cognitivamente, come nel caso di malattie degenerative o psichiatriche.

La difficoltà di fare i conti con queste perdite non dipende da fattori interni all'individuo o da problematiche interne alle famiglie, ma è generata dalla confusione di ruoli e confini che si viene a creare rispetto alla configurazione che la famiglia assume. Si tratta di normali reazioni a una situazione complessa che deve essere sostenuta con interventi mirati a fare emergere la resilienza delle famiglie (Walsh, 2008). L'irreversibile trasformazione multiculturale della nostra comunità sociale, inoltre, è già maturata al punto che, anche nel contesto italiano, le famiglie migranti fanno i conti con i loro componenti di "seconda generazione”, quelli cioè che si trovano a fronteggiare le contraddizioni emergenti sia dall'intreccio di due generazioni, sia dall'intreccio di due culture, aprendo spesso situazioni di crisi coinvolgenti tutta la famiglia. Il processo di autonomizzazione e di costruzione dell'identità dei giovani, infatti, oscilla, in questi casi, tra problematiche relazionali tipiche dei momenti di passaggio all'adultità e problematiche di collocazione culturale nel contesto di origine dei genitori e in quello di approdo. Anche questi sono processi particolarmente complessi che possono richiedere un sostegno alle famiglie a gestire le dinamiche implicate.

Infine, la diffusione dei mezzi di comunicazione digitale non è un elemento solo tecnico: essi hanno fatto irruzione nella vita delle persone, modificando i modi stessi della comunicazione interpersonale e dunque anche familiare. La possibilità di essere costantemente connessi annulla i confini, permette di mantenere rapporti con un altrove più ampio. In certi casi, i rapporti familiari sono favoriti dai nuovi media per le straordinarie opportunità connettive che essi offrono; contemporaneamente, però, nelle famiglie aumenta la preoccupazione per gli effetti di dipendenza che l'utilizzo pervasivo dei nuovi media può indurre. Nel momento in cui sto scrivendo, il nostro Paese sta affrontando la pandemia da Covid-19. Le famiglie sperimentano giorno per giorno questo tipo di oscillazioni.

I processi di sviluppo che caratterizzano le famiglie contemporanee sono complessi e per tanti versi nuovi sia per le famiglie sia per i professionisti. Tuttavia, occorre sottolineare che si tratta di processi iscritti nell'ambito della fisiologia delle famiglie caratterizzate da particolari strutture, processi fondativi o circostanze di vita, e in quanto tali devono essere trattati (Walsh, 2003; Coleman, Ganong, 2004; Fruggeri, 2005).

Le famiglie affrontano questi processi grazie alle risorse interne (personali e interpersonali, culturali ed economiche), ma anche alle risorse esterne, ovvero grazie al supporto sociale di tipo informale (amici e parenti) e quello di tipo formale (i servizi psico-socio-educativi-sanitari). I professionisti di questi servizi sono pertanto chiamati ad affiancare le famiglie nel fronteggiamento di tutti i compiti fin qui descritti, nel rispetto delle specificità che ogni tipo di famiglia rappresenta. Purtroppo, i documenti programmatici e i professionisti stessi dei servizi che accompagnano, sostengono, aiutano e curano le famiglie nei loro percorsi evolutivi, sono in larga parte ancorati a modelli di riferimento e procedure adatte a un unico tipo di famiglia, quella tradizionale autoctona, composta da coppia eterosessuale con figli biologici conviventi. Questo dato deve fare riflettere i professionisti dei servizi e richiamarli alla responsabilità di adottare, invece, modelli in grado di sostenere le fa- 
miglie per la specificità dei processi che le caratterizzano.

\section{La cultura della differenza come guida metodologica per il sostegno alle famiglie contemporanee}

L'eterogeneità delle famiglie richiede che l'intervento dei professionisti impegnati nel sostegno alle famiglie si ispiri alla cultura della differenza, ovvero che si avvalga di modelli capaci di sostenere le dinamiche e i processi della vita quotidiana di tutti i tipi di famiglie senza pregiudizi e discriminazioni. Questo implica innanzitutto abbandonare la cultura della devian$z a$ che riconduce la differenza di struttura, di formazione o di circostanze di vita alla psicopatologia. La cultura della devianza confronta ogni famiglia con il modello, ritenuto tipico-ideale, della famiglia nucleare tradizionale con coppia eterosessuale e figli biologici conviventi, di classe media, appartenente alla cultura occidentale. La prospettiva della devianza considera pregiudizialmente problematiche tutte le famiglie non corrispondenti al modello tradizionale.

La cultura della differenza, invece, parte dal presupposto, verificato in innumerevoli ricerche, che la qualità del funzionamento di tutte le famiglie sia riconducibile alla qualità dei processi, delle relazioni e delle dinamiche del gruppo con cui esse affrontano i compiti e le situazioni della loro vita quotidiana, al di là di astratti modelli di confronto. Il punto di partenza della cultura della differenza è quello di valutare il funzionamento delle famiglie sulla base della qualità dei processi e delle dinamiche relazionali attraverso cui esse fanno fronte ai loro compiti di sviluppo. Questo orientamento metodologico ha una premessa importante, ovvero che la condizione per un buon funzionamento familiare non sia l'assenza o l'evitamento di problemi o di difficoltà, bensì la capacità di fronteggiare i problemi e gli eventi critici.

Mentre la cultura della devianza contiene in sé un pregiudizio che consiste nel considerare la diversità delle famiglie dal modello tradizionale come un fattore di rischio, ovvero nel considerare la diversità come un handicap; la cultura della differenza considera la diversità familia- re espressione di specificità di funzionamento e dunque si interroga su quali siano i compiti che queste famiglie devono affrontare e quali siano i processi specifici che devono essere attivati per il benessere dei loro membri.

\section{I compiti inconsueti delle famiglie contemporanee}

Certi eventi critici che in generale le famiglie affrontano nel corso della loro vita sono parte della cultura della comunità di appartenenza, che offre vari repertori di azione per attivare le risorse necessarie a farvi fronte. Si pensi al lutto: per quanto doloroso e sconvolgente possa essere nella vita di una famiglia, esso è un evento critico definibile come consueto poiché per esso esistono repertori di azione condivisi, composti di regole, rituali e procedure che le persone possono seguire come binari contenitivi dell'incertezza che un evento critico può scatenare. Esistono invece degli eventi che alcune famiglie contemporanee devono affrontare, che possiamo definire inconsueti perché non sono ancora integrati come fatti fisiologici nella vita delle famiglie, e sono dunque privi di strategie note con cui affrontarli. Se una donna separata con figli si innamora di una persona, deve comunicarlo o no ai propri figli? Se sì, come, quando? Che ruolo va a ricoprire il nuovo partner di un genitore separato nella famiglia allargata o nel nucleo convivente? Come possono coordinarsi due persone in conflitto, legate da sentimenti negativi di rabbia e delusi nelle reciproche aspettative, per gestire la genitorialità? Cosa dire ai figli la cui procreazione è avvenuta grazie al contributo di un terzo? Che controllo deve avere un genitore sullo smartphone dei figli? Come devo interpretare il fatto che mio figlio adottivo sta cercando attraverso internet e i social network i propri genitori biologici? Come si ricompongono i familiari delle famiglie migrate per tappe? Come fa il genitore che lascia i figli al Paese di origine a mantenere la sua funzione genitoriale?

Tutte queste domande non trovano ancora sperimentati ancoraggi nella conoscenza comune. I modi di fare fronte a questi eventi non sono ancora incorporati nelle pratiche della comunità di appartenenza e in questo senso non hanno riferimenti esterni a cui affidarsi. I compiti inconsueti delle famiglie contemporanee sono affrontati nella negoziazione quotidiana, generatrice di soluzioni non contemplate a priori.

Ma i compiti inconsueti delle famiglie contemporanee sono spesso inconsueti anche per i professionisti e a questo proposito due sono gli aspetti da sottolineare la necessità /dovere di:

1 assimilare l'inconsuetudine dei compiti, eventi e processi delle famiglie contemporanee alla psicopatologia o alla devianza;

2 approfondire queste nuove tematiche rivolgendosi a quanto la ricerca scientifica ha prodotto negli ultimi anni. La letteratura mostra, infatti, come l'assunzione di una prospettiva fondata sulla conoscenza specifica dei processi attivati nelle diverse famiglie possa risultare utile a chi opera in campo clinico, psicosociale ed educativo per individuare i probabili momenti di tensione, da una parte, e attivare i processi efficaci a farvi fronte, dall'altra (Walsh, 2003).

La rubrica che inizia con questo numero si propone di fornire un aiuto proprio in questa direzione.

\section{laura.fruggeri@gmail.com}

Boss P. Ambiguous loss. Harvard University Press, 1999.

Coleman M, Ganong HL (a cura di). Handbook of contemporary families: considering the past, contemplating the future. Sage Publications, 2004.

Fruggeri L (a cura di). Famiglie d'oggi. Carocci, 2018.

Fruggeri L. Diverse normalità. Carocci, 2005. Golombok S. Famiglie Moderne. Genitori e figli nelle nuove forme di famiglia. Edra, 2016.

Golombok S. Parents: What really counts. Psychology Press, 2000.

Lingiardi V, Carone N. Madri lesbiche, padri gay: genitori de-generati? Giornale Italiano di Psicologia. 2016;1-2:57-80.

Parke RD. Future families. Different forms, rich possibilities. Wiley, 2013.

Walsh F. La resilienza familiare. Raffello Cortina, 2008.

Walsh F. Normal family processes. The Guilford Press, 2003. 